\title{
Erratum to: Potential of Plant Growth Promoting Traits by Bacteria Isolated from Heavy Metal Contaminated Soils
}

\author{
Vijay Kumar $^{1} \cdot$ Simranjeet Singh $^{2} \cdot$ Joginder Singh $^{2} \cdot$ Niraj Upadhyay $^{3}$
}

Published online: 25 April 2015

(C) Springer Science+Business Media New York 2015

\section{Erratum to: Bull Environ Contam Toxicol DOI 10.1007/s00128-015-1523-7}

There was a mistake in the presentation of the accession number of reported strains in the online published article which has been corrected now.

Please read, "16s rDNA analyses have confirmed following strains with GenBank accession numbers: Bacillus thuringiensis PS-1 (KC 355253.1), Azotobacter chroococcum PS-2 (JX913866.1), Paenibacillus ehimensis PS-4 (FN582330.1), and Pseudomonas pseudoalcaligenes PS-5
(AB109887.1)" as "16s rDNA analyses have confirmed following strains with GenBank accession numbers: Bacillus thuringiensis PS-1 (KJ511861.1), Azotobacter chroococcum PS-2 (KJ607246.1), Paenibacillus ehimensis PS-4 (KJ511862.1), and Pseudomonas pseudoalcaligenes PS-5 (KJ588061.1)".

The accession numbers were wrongly uploaded on NCIB database and the authors were unaware about it. The correct accession numbers are provided here. Also, the typological error "NICM" should read as "NCIM" throughout the article.
The online version of the original article can be found under doi:10.1007/s00128-015-1523-7.

Niraj Upadhyay

nivij.res@gmail.com

1 Department of Chemistry, Lovely Professional University, Jalandhar 144002, Punjab, India

2 Department of Biotechnology, Lovely Professional University, Jalandhar 144002, Punjab, India

3 Department of Chemistry, Dr. Hari Singh Gour University, Sagar, Madhya Pradesh, India 